\title{
Performance assessment of genetic programming (GP) and minimax probability machine regression (MPMR) for prediction of seismic ultrasonic attenuation
}

\author{
Manoj Kumar • Manav Mittal · Pijush Samui
}

Received: 15 July 2013/ Accepted: 15 August 2013/Published online: 30 October 2013

(c) The Seismological Society of China, Institute of Geophysics, China Earthquake Administration, and Springer-Verlag Berlin Heidelberg 2013

\begin{abstract}
The determination of seismic attenuation $(s)(\mathrm{dB} / \mathrm{cm})$ is a challenging task in earthquake science. This article employs genetic programming (GP) and minimax probability machine regression (MPMR) for prediction of $s$. GP is developed based on genetic algorithm. MPMR maximizes the minimum probability of future predictions being within some bound of the true regression function. Porosity (n) (\%), permeability (k) (millidarcy), grain size $(d)(\mu \mathrm{m})$, and clay content (c) (\%) have been considered as inputs of GP and MPMR. The output of GP and MPMR is $s$. The developed GP gives an equation for prediction of $s$. The results of GP and MPMR have been compared with the artificial neural network. This article gives robust models based on GP and MPMR for prediction of $s$.
\end{abstract}

Keywords Seismic attenuation - Genetic programming - Minimax probability machine regression · Artificial neural network · Prediction

\footnotetext{
M. Kumar $(\square)$

National Institute of Rock Mechanics, Kolar Gold Fields

563117, Karnataka, India

e-mail: manojgeologist84@gmail.com

M. Mittal

School of Mechanical and Building Science, VIT University,

Vellore 632014, Tamil Nadu, India

e-mail: manav.mittal2312@gmail.com

P. Samui

Centre for Disaster Mitigation and Management, VIT University,

Vellore 632014, India

e-mail: pijush.phd@gmail.com
}

\section{Introduction}

The determination of seismic attenuation $(s)(\mathrm{dB} / \mathrm{cm})$ is an imperative task in earthquake science. $s$ depends on different rock properties, for example, porosity $(n)(\%)$, permeability $(k)$ (milidarcy), grain size $(d)(\mu \mathrm{m})$, and clay content $(c)(\%)$. Researchers gave different attenuation mechanisms (Biot 1956a, b; Walsh 1966, 1969; Stoll and Bryan 1970; Solomon 1973; Kuster and Toksoz 1974; Mavko and Nur 1975, 1979). Klimentos and Mccann (1990) used regression model for prediction of $s$. Brzostowski and McMechan (1992), and Leggett et al. (1992) developed the model based on the change in seismic amplitude as observed data for prediction of $s$. Researchers also used the rise time associated with the broadening effect for determination of $s$ (Kjartansson 1979; Zucca et al. 1994). Boadu (1997) successfully used artificial neural network (ANN) for prediction of $s$. However, ANN has various limitations such as black-box approach, low generalization capability, arriving at local minima, overtraining, etc. (Park and Rilett 1999; Kecman 2001). Quan and Harris (1997) successfully adopted frequency shift method to predict $s$. Roth et al. (2000) developed an empirical equation for prediction of $s$.

This article employs genetic programming (GP) and minimax probability machine regression (MPMR) for prediction of $s$. GP is developed based on genetic algorithm (GA) (Koza 1992). It has been successfully applied for modeling different problems in engineering (Rezapour et al. 2010; Guven and Kisi 2011; Azamathulla and Zahiri 2012). MPMR is developed based on Minimax Probability Machine (MPM) (Lanckriet et al. 2002, 2003). Many applications of MPMR are available in literatures (Yang et al. 2010; Zhou et al. 2011, 2013). GP and MPMR have been developed based on the database collected from the 
Table 1 Statistical parameters of the dataset

\begin{tabular}{lcccc}
\hline Variables & Mean & Standard deviation & Skewness & Kurtosis \\
\hline$n(\%)$ & 20.62 & 8.97 & -0.06 & 2.02 \\
$k($ milidarcy) & 39.54 & 73.42 & 2.30 & 7.50 \\
$d(\mu \mathrm{m})$ & 142 & 85.18 & 1.15 & 3.11 \\
$c(\%)$ & 12.12 & 8.10 & 0.07 & 2.08 \\
$s(\mathrm{~dB} / \mathrm{cm})$ & 3.33 & 2.43 & 0.53 & 2.77 \\
\hline
\end{tabular}

Table 2 Training dataset

\begin{tabular}{|c|c|c|c|c|}
\hline$n(\%)$ & $k$ (milidarcy) & $d(\mu \mathrm{m})$ & $c(\%)$ & $s(\mathrm{~dB} / \mathrm{cm})$ \\
\hline 29.81 & 17.17 & 74 & 12 & 4.51 \\
\hline 35.98 & 21.16 & 76 & 16 & 4.54 \\
\hline 20.55 & 0.05 & 80 & 15 & 3.15 \\
\hline 2.9 & 0 & 70 & 0 & 0.08 \\
\hline 32.397 & 9.3 & 78 & 23 & 8.92 \\
\hline 35.129 & 73.26 & 82 & 30 & 8.48 \\
\hline 33.1 & 10.05 & 83 & 20 & 4.83 \\
\hline 23.42 & 11.42 & 85 & 15 & 2.4 \\
\hline 24.7 & 7.1 & 91 & 22 & 3.47 \\
\hline 29.943 & 9.59 & 84 & 17 & 5.02 \\
\hline 30.69 & 3.5 & 102 & 17 & 4.59 \\
\hline 24.84 & 1.13 & 91 & 25 & 7.68 \\
\hline 11.93 & 0.01 & 87 & 7 & 1.79 \\
\hline 15.11 & 0.06 & 74 & 14 & 4.92 \\
\hline 19.05 & 0.13 & 72 & 15 & 6.83 \\
\hline 28.83 & 10.27 & 82 & 20 & 3.33 \\
\hline 33.249 & 2.25 & 80 & 15 & 5.26 \\
\hline 27.74 & 5.78 & 87 & 20 & 4.19 \\
\hline 29.412 & 7.03 & 91 & 23 & 4.93 \\
\hline 18.645 & 2.21 & 145 & 12 & 2.68 \\
\hline 17.794 & 0.37 & 140 & 12 & 2.36 \\
\hline 14.783 & 220.9 & 242 & 0.2 & 0.08 \\
\hline 15.065 & 150.7 & 229 & 1 & 0.7 \\
\hline 14.518 & 160.4 & 260 & 0.7 & 0.14 \\
\hline 15.64 & 87.65 & 235 & 0.5 & 0.09 \\
\hline 16.514 & 41.74 & 377 & 15 & 3.63 \\
\hline 17.06 & 50.51 & 312 & 15 & 3.3 \\
\hline 12.045 & 3.67 & 226 & 7 & 2.1 \\
\hline 16.48 & 87.55 & 226 & 5 & 0.47 \\
\hline 8.21 & 0.13 & 140 & 6 & 2.46 \\
\hline 26.56 & 305.8 & 187 & 5 & 2.73 \\
\hline 11.98 & 0.46 & 153 & 6 & 1.6 \\
\hline 5.45 & 0 & 97 & 3 & 0.19 \\
\hline 9.94 & 0.16 & 97 & 9 & 4.63 \\
\hline
\end{tabular}

work of Boadu (1997). The developed GP and MPMR have been compared with the ANN model. The paper is organized as follows. The details of GP are given in Sect. 2 .
Table 3 Testing dataset

\begin{tabular}{lcccl}
\hline$n(\%)$ & $k$ (milidarcy) & $d(\mu \mathrm{m})$ & $c(\%)$ & $s(\mathrm{~dB} / \mathrm{cm})$ \\
\hline 3.489 & 0 & 78 & 0 & 0.01 \\
31.02 & 5.47 & 84 & 18 & 4.5 \\
27.57 & 0.45 & 76 & 25 & 8.61 \\
20.48 & 0.44 & 79 & 8 & 1.57 \\
28.543 & 33.67 & 139 & 15 & 4.18 \\
15.431 & 255.9 & 272 & 1 & 0.29 \\
16.03 & 52.42 & 330 & 15 & 3.38 \\
14.125 & 11.06 & 271 & 4 & 1.65 \\
\hline
\end{tabular}

Section 3 describes the details of MPMR. Section 4 gives the details of results and discussion. Major conclusions have been drawn in Sect. 5 .

\section{Details of GP}

GP predicts output based on the dataset. In GP, a random population of equation is created. In next step, fitness of each equation is determined. "Parents" are selected out of these individuals through tournament. "Offsprings" are created from "Parents" through the process of reproduction, mutation, and crossover. The details of reproduction, mutation, and crossover are given by Koza (1992). The best equation that produced any generation is the solution of the problem. $n, k, d$, and $c$ are considered as inputs of the GP. The output of GP is $s$. Training and testing dataset has been used to develop the GP model. Training dataset is used to construct the GP model. Testing dataset is adopted to verify the constructed GP model. Table 1 shows the statistical parameters of the dataset. This study uses 34 (see Table 2) datasets as training dataset. The remaining eight (see Table 3) datasets have been used as testing dataset. The program of GP has been implemented by MATLAB.

\section{Details of MPMR}

MPMR is developed by constructing a dichotomy classifier (Strohmann and Grudic 2002). MPMR adopts the following regression model for prediction of output $(y)$.

$y=\sum_{i=1}^{N} \beta_{i} K\left(x_{i}, x\right)+b$,

where $y$ is output, $x$ is input, $K\left(x_{\mathrm{i}}, x\right)$ is kernel function, and $\beta, b$ are outputs of the MPMR algorithm. This study uses $c$, $n, k$, and $d$ as inputs. The output of MPMR is $s$. So, 
$x=[c, n, k, d]$ and $y=[s]$.

MPMR creates two classes $\left(u_{\mathrm{i}}\right.$ and $\left.v_{\mathrm{i}}\right)$ of data. The expression of $u_{\mathrm{i}}$ and $v_{\mathrm{i}}$ is given below.

$u_{i}=\left(y_{i}+\varepsilon, x_{i 1}, x_{i 2}, \ldots, x_{i d}\right)$,

$v_{i}=\left(y_{i}-\varepsilon, x_{i 1}, x_{i 2}, \ldots, x_{i d}\right)$,

where $i=1, \ldots, N$.

The classification boundary between $u_{\mathrm{i}}$ and $v_{\mathrm{i}}$ is a regression surface. MPMR uses the same training and testing dataset as used by the GP. Radial basis function $\left(K\left(x_{i}, x\right)=\exp \left[-\frac{\left(x_{i}-x\right)\left(x_{i}-x\right)^{T}}{2 \sigma^{2}}\right]\right.$ where $\sigma$ is width of radial basis function) has been used as kernel function. The data is normalized between 0 and 1 . The program of MPMR has been constructed by using MATLAB.

\section{Results and discussion}

For GP, the number of population is set to 800 . The number of generation is kept to 100 . The mutation frequency and crossover frequency is kept to 70 and 50, respectively. Figures 1 and 2 illustrates the performance of training and testing, respectively. The performance of GP has been assessed in terms of coefficient of correlation $(R)$ value. For a good model, the value of $R$ is close to one. The value of $R$ has been shown in Figs. 1 and 2. The developed GP gives the following expression for determination of $s$.

$$
\begin{aligned}
s= & 5.89 c-10.61 n+0.23 \exp (n-d)+4.26 \exp (n-c) \\
& +48.19 \exp (\exp (n))-61.18\{\exp (n)\}^{2}-10.4(n)^{4} \\
& -39.24\left\{\exp (n)^{2}\right\}^{2}+61.81 c^{2}-34.52
\end{aligned}
$$

Figures 1 and 2 shows that the value of $R$ is close to one. So, the developed GP predicts $s$ reasonably well.

For MPMR, the design values of $\sigma$ and $\varepsilon$ have been determined by trial and error approach. The developed MPMR gives the best performance at $\sigma=0.5$ and $\varepsilon=0.003$. So, the design values of $\sigma$ and $\varepsilon$ is 0.5 and 0.003 , respectively. The performance of training and testing has been shown in Figs. 1 and 2. The value of $R$ is close to one for training as well as testing dataset. Therefore, the developed MPMR proves his capability for prediction.

The developed GP and MPMR have been compared with the ANN model developed by Boadu (1997). Comparison has been done in terms of standard error. Figure 3 shows the bar chart of standard error of the GP, MPMR, and ANN models. The developed GP and MPMR outperform the ANN model. The performance of GP and MPMR is almost same. The developed MPMR has control over future prediction. However, ANN and GP have no control over future prediction.

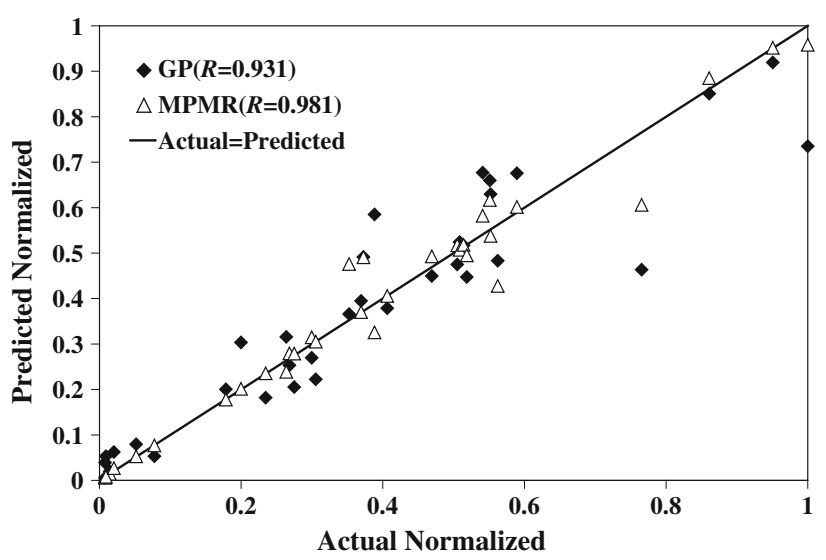

Fig. 1 Performance of training dataset

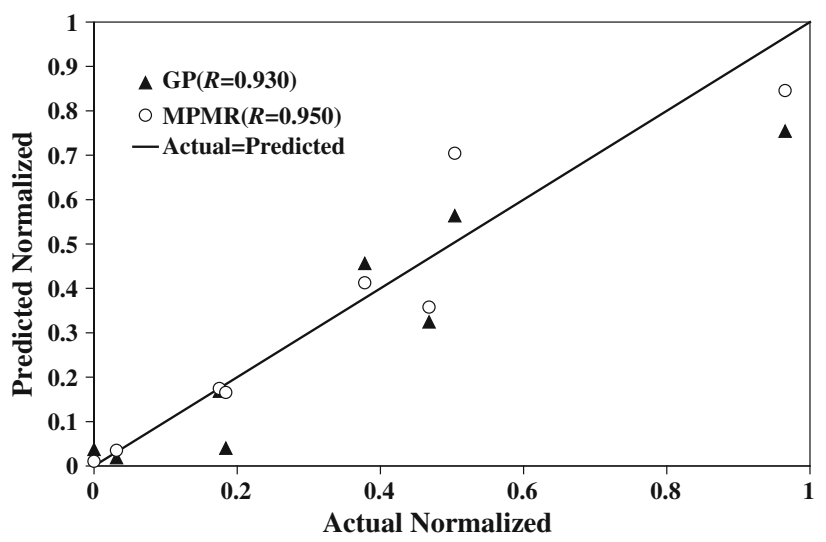

Fig. 2 Performance of testing dataset

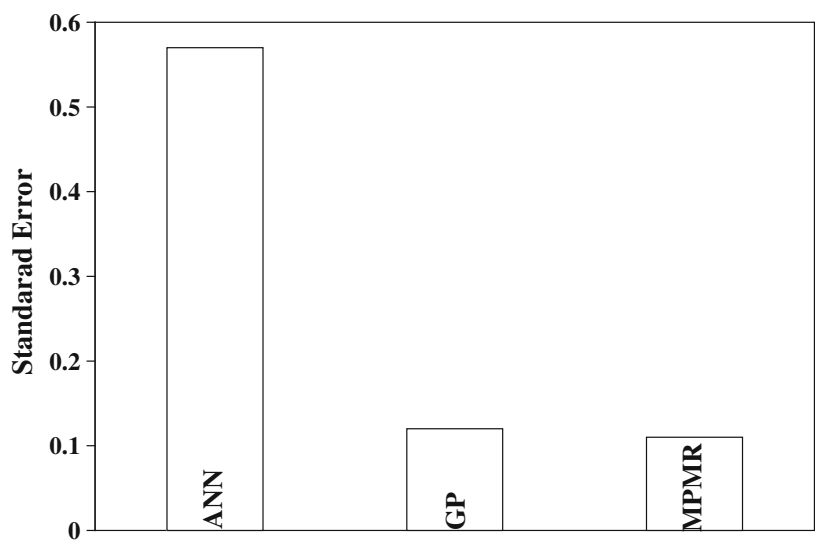

Fig. 3 Bar chart of standard error for ANN, GP, and MPMR models

\section{Conclusions}

This article describes GP and MPMR for prediction of seismic attenuation $(s)$. The developed GP and MPMR uses $n, c, k$, and $d$ as inputs. Training and testing datasets have been used to develop the GP and MPMR models. The 
predictive capability of GP and MPMR has been assessed in terms of $R$ value. The performance of GP and MPMR is encouraging. The developed GP gives an equation for prediction of $s$. The performance of GP and MPMR is better than the ANN model. The results confirm that the developed GP and MPMR show excellent predictive accuracy.

\section{References}

Azamathulla HM, Zahiri A (2012) Flow discharge prediction in compound channels using linear genetic programming. J Hydrol 454-455:203-207

Biot MA (1956a) Theory of propagation of elastic waves in a fluidsaturated porous solid. II. Low frequency range. J Acoust Soc Am 28(10):169-178

Biot MA (1956b) Theory of propagation of elastic waves in a fluidsaturated porous solid. II. High frequency range. J Acoust Soc Am 28:179-181

Boadu FK (1997) Rock properties and seismic attenuation: neural network analysis. Pure Appl Geophys 149:507-524

Brzostowski M, McMechan G (1992) 3-D tomographic imaging of near-surface seismic velocity and attenuation. Geophysics 57:396-403

Guven A, Kişi O (2011) Daily pan evaporation modeling using linear genetic programming technique. Irrig Sci 29(2):135-145

Kecman V (2001) Learning and soft computing: Support vector machines, neural networks, and fuzzy logic models. MIT Press, Cambridge

Kjartansson E (1979) Constant Q-wave propagation and attenuation. J Geophys Res 84:4137-4748

Klimentos T, McCann C (1990) Relationships among compressional wave attenuation, porosity, clay content and permeability in sandstones. Geophysics 55:998-1014

Koza JR (1992) Genetic programming: on the programming of computers by means of natural selection. MIT Press, Cambridge

Kuster GT, Toksoz MN (1974) Velocity and attenuation of seismic waves in two phase media, Part 1: Theoretical formulations. Geophysics 39:587-606

Lanckriet GRG, El Ghaoui L, Bhattacharyya C, Jordan MI (2002) Minimax probability machine. In: Proceedings of advances in neural information processing systems. MIT Press, Cambridge
Lanckriet GRG, El Ghaoui L, Bhattacharyya C, Jordan MI (2003) A robust minimax approach to classification. J Mach Learn Res 3:552-582

Leggett M, Goulty NR, Kragh JE (1992) Study of travel time and amplitude time-lapse tomography using physical model data. In: Abstracts of 54th EAEG meeting, pp 248-249

Mavko GM, Nur A (1975) Melt squirt in the asthenosphere. J Geophys Res 80:1444-1448

Mavko GM, Nur A (1979) Wave attenuation in partially saturated rocks. Geophysics 44:161-178

Park D, Rilett LR (1999) Forecasting freeway link ravel times with a multi-layer feed forward neural network. Comput Aided Civil Infastruct Eng 14:358-367

Quan Y, Harris JM (1997) Seismic attenuation tomography using the frequency shift method. Geophysics 62(3):895-905

Rezapour OM, Shui LT, Dehghani AA (2010) Review of genetic programing in water resource engineering. Aust J Basic Appl Sci 4(11):5663-5667

Roth DA, Erich G, Wiens DZ (2000) An empirical relationship between seismic attenuation and velocity anomalies in the upper mantle. Geophys Res Lett 27:601-604

Solomon SC (1973) Shear wave attenuation and melting beneath the mid-Atlantic ridge. J Geophys Res 78:6044-6059

Stoll RD, Bryan GM (1970) Wave attenuation in saturated sediments. J Acoust Soc Am 47:1440-1447

Strohmann TR, Grudic GZ (2002) A formulation for minimax probability machine regression. In: Dietterich TG, Becker S, Ghahramani Z (eds) Advances in neural information processing systems (NIPS) 14. MIT Press, Cambridge

Walsh JB (1966) Seismic wave attenuation in rock due to friction. J Geophys Res 84:3532-3536

Walsh J (1969) New analysis of attenuation in partially melted rock. J Geophys Res 74:4333-4337

Yang L, Wang L, Sun Y, Zhang R (2010) Simultaneous feature selection and classification via Minimax Probability Machine. Int J Comput Intell Syst 3(6):754-760

Zhou Y, Wang R, Xia K (2011) Nonlinear prediction of fast fading channel based on minimax probability machine. In: Proceedings of the 2011 6th IEEE conference on industrial electronics and applications, ICIEA 5975626, pp 451-454

Zhou Z, Wang Z, Sun X (2013) Face recognition based on optimal kernel minimax probability machine. J Theor Appl Inf Technol 48(3):1645-1651

Zucca JJ, Hutchings LJ, Kasameyer PW (1994) Seismic velocity and attenuation structure of the Geysers geothermal field, California. Geothermics 23:111-126 